American Journal of Pharmaceutical Education 2020; 84 (4) Article 7415.

\title{
RESEARCH
}

\section{A Pilot Interprofessional Course on Substance Use Disorders to Improve Students' Empathy and Counseling Skills}

\author{
Andrew Muzyk, PharmD, MHPE, ${ }^{\mathrm{a}}$ Patricia Mullan, PhD, ${ }^{\mathrm{b}}$ Kathryn Andolsek, MD, MPH, ${ }^{\mathrm{c}}$ \\ Anne Derouin, DNP, ${ }^{\mathrm{d}}$ Zach Smothers, $\mathrm{MSc},{ }^{\mathrm{c}}$ Charles Sanders MAPPCP, ${ }^{\mathrm{d}}$ Shelley Holmer, $\mathrm{MD}^{\mathrm{c}}$ \\ ${ }^{a}$ Campbell University College of Pharmacy and Health Sciences, Buies Creek, North Carolina \\ ${ }^{\mathrm{b}}$ University of Michigan, Learning Health Science Division of Professional Education, Ann Arbor, Michigan \\ ${ }^{c}$ Duke University, School of Medicine, Durham, North Carolina \\ ${ }^{\mathrm{d}}$ Duke University, School of Nursing, Durham, North Carolina \\ Submitted October 10, 2018; accepted August 7, 2019; published April 2020.
}

Objective. To implement and assess the effectiveness of an interprofessional course designed to provide pharmacy and nursing students with opportunities to improve their understanding of substance use disorders (SUDs), assess their attitudes toward patients with SUDs, receive instruction and feedback on behavior change counseling, and engage in interprofessional education.

Methods. The course consisted of four interactive class sessions that focused on empathy and recognition of personal bias, behavioral change counseling, and SUD recognition, screening, and treatment. Classes were taught by an interprofessional faculty member. Students also attended a 12-step recovery meeting in the community, counseled an authentic patient using behavioral change counseling, and received feedback based on a faculty member's direct observation of their counseling. Students completed validated assessments of their attitudes toward patients with SUDs and interprofessional collaboration. A faculty member used the Behavioral Change Counseling Index (BECCI) to assess students' counseling of SUD patients. Counseled patients were asked their preference for follow-up care for their SUD.

Results. Twenty-seven pharmacy and nursing students completed this course, along with 51 students from other professions. The pharmacy and nursing students demonstrated significant improvements in their attitudes toward patients with SUDs and toward interprofessional collaboration, as measured by the Substance Abuse Attitudinal Scale (SAAS) and Student Perception of Interprofessional Clinical Education (SPICE-R2). Approximately $93 \%$ of pharmacy and nursing students counseled a patient with a SUD, with $96 \%$ of counseled patients reporting their intent to receive follow-up care.

Conclusion. An interprofessional SUD course enriched pharmacy and nursing students' understanding, attitudes, and behaviors toward patients with SUDs and interprofessional collaboration. Students demonstrated an ability to provide behavior change counseling to patients, and $96 \%$ of counseled patients stated a willingness for follow-up care for their SUD.

Keywords: interprofessional, health professions, addiction, counseling, opioid

\section{INTRODUCTION}

As of 2016, approximately 20.1 million people in the United States aged 12 years or older had a substance use disorder (SUD), which contributed to more than 42,000 deaths from opioids in that year alone. ${ }^{1,2}$ Health care professionals from multiple disciplines provide care to patients with SUDs. Notably, the role of pharmacists and nurses in this area has increased. ${ }^{3,4}$ Pharmacy and nursing organizations have advocated for increased education

Corresponding Author: Andrew J. Muzyk, Campbell University College of Pharmacy and Health Sciences, Buies Creek, NC. Email: Andrew.Muzyk@duke.edu. and training in SUDs to better prepare these clinicians and to expand their skills in collaborative patient care. ${ }^{5-9}$ Educational organizations have similarly recognized the need for improving curricular content on SUDs to prepare future health care professionals, increase patients' access to effective health care, and reduce the stigma of SUDs. The converging public and professional concerns about the opioid crisis in the United States heightens the need for health professions schools to make timely efforts to provide education in this area to their students. ${ }^{10}$

A report from the American Association of Colleges of Pharmacy (AACP), entitled "Curricular Guidelines for Pharmacy Education: Substance Abuse and Addictive 


\section{American Journal of Pharmaceutical Education 2020; 84 (4) Article 7415.}

Disease," recommends that pharmacy students should have numerous opportunities to learn about the identification and treatment of SUDs, the psychosocial aspects of care, and first-person community-based experiences, such as visiting meetings for Alcoholics Anonymous or similar 12-step programs. ${ }^{11}$ The American Nurses Association (ANA) encourages that comprehensive nursing education across all levels of professional preparation include topics on the misuse, abuse, and addictive potential of prescription drugs, as well as the vulnerabilities of special populations, which may increase their risk for drug dependence. ${ }^{9}$ Additonally, the American Association of Colleges of Nursing (AACN) recommends all nursing schools adopt an enhanced curriculum to address the opioid epidemic. ${ }^{12}$

Two systematic reviews of substance use education in both pharmacy and nursing schools conducted in 2017 reported a paucity of SUD educational interventions for pharmacy or nursing students. ${ }^{13,14}$ The majority of reports related to SUD education described interventions and assessments lacking validity evidence, which limited study rigor, generalizability of findings, and reproducibility of the interventions described. The studies primarily focused on tobacco and alcohol use disorders and evaluated students' knowledge or attitudinal changes. None of the studies described student interactions with authentic patients or outcomes related to changing student or patient behaviors. Lastly, very few studies involved students from more than one health professions program.

We developed an interprofessional SUD course that combined classroom learning with direct application to patient care in the clinic setting. We intended to move health professions students' learning to action-oriented educational outcomes including provider behavior and patient outcomes. ${ }^{15,16}$ The objective of this course was to provide pharmacy and nursing students with opportunities to: improve their understanding of SUDs, assessstudent attitudes toward patients with SUDs; develop skills in patient communication and provide actionablefeedback on their behavior change counseling session while learning and from other health professions students.This paper reports on research findings from the experience of pharmacy and nursing students who participated in this innovative course.

\section{METHODS}

Beginning in the spring 2018 semester, health professions students completing a psychiatry clerkship within the Duke University Health System participated in an interprofessional SUD course. Students came from medicine, physician assistant studies, pharmacy, social work, and accelerated Bachelors of Science in Nursing
(ABSN) programs. While the course was an elective for the ABSN students, it was required for all other students. The course was repeated monthly over five months with approximately 15 health professions students in each of the five cohorts. The experiences and outcomes of all of the health professions students are described in a separate paper. ${ }^{17}$

All pharmacy and nursing students participated in four interactive, in-person classes that lasted approximately 90 minutes each and focused on SUDs. The students also attended one community-based 12-step recovery meeting and wrote a short reflection paper about their experience, and conducted behavioral change counseling with a patient in a clinical setting.

To facilitate a holistic approach to SUD learning, we designed classes focused on developing the following: empathy and recognition of personal bias, skills in behavioral change counseling, and knowledge of SUD recognition, screening, and treatment. Classes were facilitated by an interprofessional group of faculty members. All class sessions used an active-learning approach that included Socratic questioning, case-based learning, role-playing, or guided reflection. Therapeutic cases were used to engage students in their learning, with cases adapted from multiple sources, including MedEd Portal (https://www. mededportal.org/), relevant textbooks, peer-reviewed articles, and the study authors' clinical experience.

Following the class session on behavior change counseling, pharmacy and nursing students had an opportunity to counsel a patient with a SUD in the clinical setting using behavioral change counseling. A focus was placed on selecting patients that were Patients werer chosen so that they were medically and psychiatrically stable enough to participate in counseling and able to provide verbal consent. A study team member who was experienced in behavior change counseling, provided observation and assessment of and feedback on students' behavioral change counseling.

As part of their patient counseling, students asked patients about their willingness to participate in postdischarge follow-up regarding their SUD treatment. The follow-up options included: agreeing to start a medication for his/her SUD, calling the North Carolina Quitline for smoking cessation, scheduling an outpatient referral with an addiction care provider, being open to having future discussions about his/her SUD, or not wanting to further discuss his/her SUD. Students communicated this information back to the patients' treatment team. We reported this "patient response" to capture each patient's decision regarding follow-up care for his/her SUD.

Assessments of pharmacy and nursing students' learning included measures of their attitudes toward SUD 


\section{American Journal of Pharmaceutical Education 2020; 84 (4) Article 7415.}

and toward interprofessional collaboration, and their performance of behavioral change counseling. Students' attitudes toward patients with SUDs were measured on the Substance Abuse Attitude Scale (SAAS) and their attitudes toward interprofessional education and practice were measured on the Students Perception of Interprofessional Clinical Education, Version 2 (SPICE-R2). ${ }^{18,19}$ Students completed these assessments at the end of the course using a retrospective pre-post approach in which, for each survey question, students first characterized their current self-perception (post-course) then characterized their attitude prior to starting the course (pre-course) ${ }^{20} \mathrm{~A}$ faculty member experienced in behavior change counseling assessed students' observed performance of behavioral change counseling of patients using the Behavior Change Counseling Index (BECCI). ${ }^{21}$ Study outcomes included: nursing and pharmacy students' attitudes toward substance abuse as assessed by pre- and post-course SAAS scores; students' attitudes toward interprofessional education and care as assessed by comparing pre- and post-course SPICE-R2 scores; students' observed performance conducting behavioral change patient counseling as assessed using BECCI ratings; and patients' reported willingness to accept treatment following student-led behavioral change counseling.

The SAAS assessment scale contains five domains comprised of 25 questions with five Likert-type responses ranging from $1=$ strongly disagree to $5=$ strongly agree. ${ }^{18}$ The five domains include permissiveness, non-stereotyping, treatment intervention, treatment optimism, and non-moralism. The SAAS gas previously been validated and shown to reliably assess students' attitudes toward patients with SUDs.

The SPICE-R2 assessment scale contains three domains comprised of 10 questions. ${ }^{19}$ The three domains are interprofessional teamwork and team-based practice, roles/responsibilities for collaborative practice, and patient outcomes from collaborative practice. The 10 questions on the SPICE-R2 contain Likert-type responses ranging from $1=$ strongly disagree to $5=$ strongly agree . The SPICE-R2 instrument has demonstrated reliability and construct validity.

The BECCI instrument scores individuals' competency in using behavior change patient counseling. ${ }^{21}$ The 11 -items on the BECCI scale responses range from $0=$ not at all to $4=$ a great extent. One additional question asks the observer to estimate the approximate amount of time the student practitioner spent speaking, selecting from one of three choices: more than half the time, about half the time, and less than half the time. The BECCI instrument has demonstrated reliability, validity, and responsiveness to educational interventions.
Students voluntarily completed the SAAS and SPICE-R2 surveys online. A consent form accompanied the survey instruments. The BECCI instrument was completed online by the faculty member directly observing the students' behavioral change counseling. Data from the three (SAAS, SPICE-R2, and BEECI) assessment measures were managed through Duke Office of Clinical Research using REDCap (Research Electronic Data Capture), which is a secure web-based application. ${ }^{22}$ Students received no incentive for completing the surveys, and the course director who determined the students' grades was not aware of whether a student completed the surveys. The research was deemed exempt by the Duke Institutional Review Board.

Descriptive statistics calculated for the SAAS, SPICE-R2, and BECCI scores included the mean, standard deviation, median, and $25^{\text {th }}$ and $75^{\text {th }}$ percentiles. The mean difference (post-course score minus pre-course score) was evaluated for the SAAS and SPICE-R2 assesments using a paired $t$ test; a $p$ value $<.05$ was considered significant. The effect size for the SAAS and SPICE-R2 were computed as the mean difference divided by the standard deviation of the differences. Student $t$ test, with equal variance assumed, was used to compare the attitudinal SAAS and SPICE-R2 scores between pharmacy and nursing students. This analysis was conducted to determine if one group was influencing the overall study findings. Categorical data are expressed as the raw number obtained as well as the percentage for non-missing data. All analyses were done using SAS, version 9.4 (SAS, Inc., Institute, Cary, NC.).

\section{RESULTS}

Of the 78 health professions students who completed one of the five offerings of the course over the five-month study period, 13 were pharmacy students and 14 were nursing students. Eleven pharmacy and 14 nursing students completed the study outcome measures for an overall response rate of 25 of 27 (93\%). Two students (7\%) did not complete the attitudinal surveys, and two students $(7 \%)$ did not complete the behavior change counseling session. Two (8\%) of the 25 patients' responses regarding their willingness for follow-up care for their SUD were not captured.

Pharmacy and nursing students' attitudinal measure responses are reported in Tables 1 and 2. Students' observed performance in counseling a SUD patient is reported in Table 3. Students' attitudes toward patients with substance use significantly improved following their participation in our course, as measured by overall SAAS, as well as the individual domains of non-moralizing, treatment optimism, treatment intervention, and non-stereotyping 


\section{American Journal of Pharmaceutical Education 2020; 84 (4) Article 7415.}

Table 1. Pharmacy and Nursing Students' Attitudes Toward Patients With Substance Use Measured by the SAAS ${ }^{\mathrm{a}}(\mathrm{n}=25)$

\begin{tabular}{|c|c|c|c|c|c|}
\hline Domain & Pre-Course & Post-Course & Difference (Post - Pre) & $p$ value $^{\mathrm{b}}$ & Effect Size $^{c}$ \\
\hline \multicolumn{6}{|l|}{ Total score } \\
\hline Mean (SD) & $82.1(8.6)$ & $86.2(7.1)$ & $4.1(6.0)$ & & \\
\hline \multicolumn{6}{|l|}{ Non-moralizing } \\
\hline Mean (SD) & $18.8(3.8)$ & $20.4(3.3)$ & $1.6(2.9)$ & & \\
\hline Median (lower, upper quartile) & $19(16,21)$ & $21(19,23)$ & $1(0,4)$ & .01 & .54 \\
\hline Mean (SD) & $20.6(3.0)$ & $22.0(2.9)$ & $1.4(3.1)$ & & \\
\hline Median (lower, upper quartile) & $21(19,22)$ & $23(20,24)$ & $1(0,3)$ & .03 & .45 \\
\hline \multicolumn{6}{|l|}{ Treatment intervention } \\
\hline Mean (SD) & $19.0(2.2)$ & $19.8(2.7)$ & $.7(1.3)$ & & \\
\hline Median (lower, upper quartile) & $20(18,21)$ & $20(19,21)$ & $0(0,1)$ & .01 & .54 \\
\hline \multicolumn{6}{|l|}{ Non-stereotyping } \\
\hline Mean (SD) & $12.3(3.8)$ & $11.8(4.3)$ & $-.5(2.4)$ & & \\
\hline Median (lower, upper quartile) & $12(10,13)$ & $12.0(10,14)$ & $0(-2,0)$ & .32 & N/A \\
\hline
\end{tabular}

${ }^{a}$ The SAAS included 25 questions, with answers given on a five-point Likert scale, where $1=$ strongly disagree and $5=$ strongly agree

Each of five domains contain a series of questions; non-moralizing, treatment optimism, and permissive domains contain five questions; treatment intervention six questions; non-stereotyping three questions; and one question was not scored and did not belong to a domain

${ }^{\mathrm{b}} \mathrm{t}$-test was used to determine significance, defined as $\mathrm{p}<.05$, for pre- to post-course difference

${ }^{\mathrm{c}}$ Effect size was calculated using mean difference divided by the SD of the difference

(Table 1). Effect sizes across the total score and these four domains were moderate to approaching large (range $d=.45$ to .69). Only in the domain of permissiveness did the change in student responses not reach the level of significance. No significant difference in mean change was found on any SAAS item comparing pharmacy and nursing students' scores.
Students' attitudes toward interprofessional education and practice as measured with the SPICE-R2 instrument are summarized in Table 2. Students' scores on the overall SPICE-R2 measure significantly improved, as did scores on interprofessional roles and responsibilities. In contrast, no significant differences after course completion were seen in students' attitudes toward individual

Table 2. Pharmacy and Nursing Students' Attitudes Toward Interprofessional Collaboration on the SPICE-R2, ${ }^{\text {a }}$ Pre- and Post-Course $(n=25)$

\begin{tabular}{|c|c|c|c|c|c|}
\hline Domain & Pre-Course & Post-Course & Difference (Post - Pre) & $p$ Value $^{\mathrm{b}}$ & Effect Size $^{\mathrm{c}}$ \\
\hline \multicolumn{6}{|l|}{$\overline{\text { Total }}$} \\
\hline Mean (SD) & $42.3(5.4)$ & $45.0(5.1)$ & $2.5(5.1)$ & & \\
\hline Median (lower, upper quartile) & $43(39,47)$ & $47(42,49)$ & $1(0,7)$ & .02 & .50 \\
\hline \multicolumn{6}{|l|}{ Interprofessional teamwork } \\
\hline Mean (SD) & $17.8(2.5)$ & $18.7(2.3)$ & $.9(2.3)$ & & \\
\hline Median (lower, upper quartile) & $18(16,20)$ & $20(19,20)$ & $0(0,2)$ & .06 & N/A \\
\hline \multicolumn{6}{|l|}{ Roles/Responsibilities } \\
\hline Mean (SD) & $11.4(2.5)$ & $12.6(2.1)$ & $1.2(2.1)$ & & \\
\hline Median (lower, upper quartile) & $12(9,14)$ & $13(12,14)$ & $1(0,2)$ & .01 & .56 \\
\hline \multicolumn{6}{|l|}{ Patient outcomes } \\
\hline Mean (SD) & $13.3(1.5)$ & 13.7 (1.6) & $.4(1.6)$ & & \\
\hline Median (lower, upper quartile) & $13(12,15)$ & $14(13,15)$ & $0(0,2)$ & .22 & N/A \\
\hline
\end{tabular}

${ }^{a}$ The SPICE-R2 instrument includes 10 items on interprofessional teamwork and team-based practice, roles/responsibilities for collaborative practice, and patient outcomes from collaborative practice. Responses on a five-point Likert scale range from $1=$ strongly disagree to $5=$ strongly agree

${ }^{\mathrm{b}}$ t-test was used to determine significance, defined as $\mathrm{p}<.05$, for pre- to post-course difference

${ }^{\mathrm{c}}$ Effect size was calculated using mean difference divided by the SD of the difference 


\section{American Journal of Pharmaceutical Education 2020; 84 (4) Article 7415.}

Table 3. Pharmacy and Nursing Students' Observed Behavior Change Counseling Measured by the BECCI $(\mathrm{n}=25)$

\begin{tabular}{|c|c|c|}
\hline Individual Questions. The practitioner. & Mean (SD) & $\begin{array}{c}\text { Median } \\
\text { (Lower, Upper Quartile) } \\
\end{array}$ \\
\hline invites patient to talk about behavior change & $2.9(.7)$ & $3(2,3)$ \\
\hline demonstrates sensitivity to talking about other issues & $2.8(.4)$ & $3(3,3)$ \\
\hline encourages patient to talk about current behavior or status quo & $2.5(.8)$ & $2(2,3)$ \\
\hline encourages patient to talk about change & $2.3(.7)$ & $2(2,3)$ \\
\hline asks questions to elicit how patient thinks and feels about the topic & $1.8(.8)$ & $2(1,2)$ \\
\hline uses empathic listening statements when the patient talks about the topic & $2.1(.8)$ & $2(2,3)$ \\
\hline uses summaries to bring together what the patient says about the topic & $1.6(1.0)$ & $1(1,2)$ \\
\hline acknowledges challenges about behavior change that the patient faces & $1.9(1.0)$ & $2(1,3)$ \\
\hline provides information that is sensitive to patient's concerns and understanding & $2.2(.9)$ & $2(2,3)$ \\
\hline actively conveys respect for patient's choice about behavior change & $2.9(.5)$ & $3(3,3)$ \\
\hline $\begin{array}{l}\text { and patient exchange ideas about how the patient could } \\
\text { change current behavior }\end{array}$ & $1.6(1.2)$ & $2(1,2)$ \\
\hline BECCI practitioner total score & $2.3(.6)$ & $2.3(1.8,2.5)$ \\
\hline
\end{tabular}

a BECCI instrument includes 11 questions, with answers given on a five-point scale, ranging from $0=$ not at all to $4=\mathrm{a}$ great extent

domains of interprofessional teamwork and team-based practice and patient outcomes from collaborative care. Effect sizes for the total score and for roles and responsibilities for collaborative practice were moderate (range of Cohen's $d=.49$ to .56 ). No difference in mean change was found on any SPICE-R2 survey item comparing pharmacy and nursing students' scores.

Twenty-five $(93 \%)$ of the pharmacy and nursing students conducted behavior change counseling with a patient. The majority of patients had a SUD primarilyinvolving tobacco, alcohol, or opioids. As indicated in Table 3, students who participated in our course demonstrated their ability, to some extent, to: perform behavioral change counseling, including inviting the patient to talk about behavior change; convey repect for the patient's choice about behavioral change; demonstrate sensitivity in talking about other issues; encourage patients to talk about their current behavior and change; provide information sensitive to patients' concerns and understanding; and use empathic listening statements. Sixteen students (64\%) spoke for "less than half the time" allowed for the counseling session, while eight students (32\%) spoke for "about half the time," as characterized by the faculty expert observer. Only one student (4\%) spoke for "more than half the time."

Twenty-three (92\%) of the 25 counseled patients shared their intentions about follow-up care. Twenty-two (96\%) patients identified a willingness to have follow-up care for their SUDs. Nine patients (39\%) agreed to have future discussions about their SUD, while seven patients $(30 \%)$ agreed to schedule an outpatient referral with an addiction care provider. Five patients $(22 \%)$ indicated they would call the Quitline for smoking cessation management. One patient (4\%) indicated they planned to start drug therapy for their SUD. Only one patient (4\%) reported they did not wish to continue discussion related ot their SUD.

\section{DISCUSSION}

We developed an interprofessional SUD course that challenged pharmacy and nursing students' existing knowledge and attitudes about SUDs and about interprofessional collaboration. Our SUD course demonstrated the benefit of pharmacy and nursing students participating in in-person classes focused on SUDs and behavioral counseling, attending a community-based 12-step recovery meeting, and practicing behavioral change counseling with an authentic patient. The understanding, attitudes, and behaviors of pharmacy and nursing students toward patients with SUDs improved. Patient outcomes associated with pharmacy and nursing students' therapeutic communication included patients' reported intention to engage in follow-up behavioral counseling. Educational outcomes associated with this course demonstrated that classroom learning can enhance not only students' selfperceived knowledge and attitudes, but also the important educational outcomes of students' behavior and patient outcomes.

Pharmacy and nursing students in our course were expected to engage with their learning environment; participate in classroom discussions; learn with, from, and about peers, faculty members, and patients; and counsel patients using behavior change counseling methods. Students' positive attitudinal changes toward working with patients with SUDs supports our intentional classroom discussions on important topics such as stigma, personal bias, benefits of treatment interventions, and engaging patients in their care decisions. The permissive domain on 


\section{American Journal of Pharmaceutical Education 2020; 84 (4) Article 7415.}

the SAAS did not demonstrate a significant change; we feel this result may have been due to the survey questions within this domain related to marijuana use, abstinence, and Alcoholics Anonymous being essential to a person's recovery. Our course did not address marijuana use. Abstinence and Alcoholics Anonymous support groups were discussed along with other recovery goals, such as reduction in heavy drinking, and care resources, such as behavioral therapy.

Active-learning exercises, led by students and facilitated by faculty members, created an environment in which students shared their learning, beliefs, and personal stories. Students' positive attitudinal changes toward interprofessional collaboration suggest this led to personal growth and group cohesiveness. Two domains, interprofessional teamwork and team-based practice and patient outcomes from collaborative practice, increased from baseline scores but did not reach statistical significance. Future research will continue to explore ways to improve students' learning and experience toward these comprehensive domains.

Twenty-five pharmacy and nursing students received feedback on their behavior change counseling skills from a faculty member, which provided them with a foundation for continued refinement of their patient counseling skills. On 10 of the 11 items on the BECCI scale, students' behavior change counseling skills were rated "to some extent" or "a good deal," which correspond with the numerical scores of " 2 " and " 3 " (respectively) on the BECCI scale. This provides increased validity to the instruments used, as median numerical scores of the students largely reflected their comments. When providing counseling to a patient, the majority of students spoke for "less than half the time" or "about half the time," and approximately $96 \%$ of patients indicated a willingness to receive follow-up care. These findings demonstrate students' ability to engage a patient in a discussion about behavior change, to actively listen to a patient's story, and to effectively help a patient decide on follow-up care for SUD treatment. Results from the BECCI survey support students' learning from the three class sessions on empathy, examining personal bias, and behavior change counseling.

This course directly addressed the needs expressed by pharmacy and nursing organizations to provide students with opportunities for identifying, diagnosing, and treating SUDs, have first-person health care experiences, and gain appreciation for the psychosocial aspects of SUDs. We intentionally included students and faculty members from the fields of nursing and pharmacy to represent health professions involved in delivering health care to patients with SUDs. Faculty members were diligent about including students representing each of the health professions in classroom discussions and modeled collaboration. Our course also addressed the value of interprofessional SUD education helping prepare students for future collaborative care.

Limitations of our study include involvement of only one academic year of cohorts at a single institution. The cohorts that were enrolled were very small in number. We did not assess students' baseline knowledge of SUDs or their baseline skills in behavior change counseling. Additonally, patients' records were not reviewed to examine if patient's followed through on seeking SUD resources.

\section{CONCLUSION}

We developed an interprofessional SUD course that enriched pharmacy and nursing students' understanding, attitudes, and behaviors toward patients with SUDs and toward interprofessional collaboration. We established the application of classroom learning directly to patient care, and elevated students' educational outcomes. Pharmacy and nursing students gained experience counseling patients with SUDs. Students demonstrated an ability to provide behavior change counseling to patients, and $96 \%$ of counseled patients stated a willingness to receive follow-up care for their SUD.

\section{REFERENCES}

1. Center for Behavioral Health Statistics. Key Substance Use and Mental Health Indicators in the United States: Results from the 2016 National Survey on Drug Use and Health. Veterans and Military Families. SAMHSA - Substance Abuse and Mental Health Services Administration. https:/www.samhsa.gov/data/sites/default/files/ NSDUH-FFR1-2016/NSDUH-FFR1-2016.htm\#sud. Accessed March 23, 2020.

2. Centers for Disease Control and Prevention. Opioid Overdose. https://www.cdc.gov/drugoverdose/index. Accessed March 23, 2020 .

3. Jukiewicz DA, Alhofaian A, Thompson Z, Gary FA. Reviewing opioid use, monitoring, and legislature: nursing perspectives. International Journal of Nursing Sciences. 2017;4(4):430-436.

4. Reynolds V, Causey H, Mckee J, Reinstein V, Muzyk A. The role of pharmacists in the opioid epidemic. North Carolina Medical Journal. 2017;78(3):202-205.

5. NCPO Opioid Statement. NCPO Statement on Combating the Opioid Crisis. https://nabp.pharmacy/wp-content/uploads/2018/02/ NCPO-Opioid-Statement-January-2018.pdf. Accessed March 23, 2020 .

6. ASHP Statement on the Pharmacists Role in Substance Abuse Prevention, Education, and Assistance. Am J Health Syst Pharm. 2016;73(9). doi:10.2146/ajhp150542.

7. 2016 Policy Manual - American Pharmacists Association. J Am Pharm Assoc. 2016;56(4):370.

8. American Association of Colleges of Nursing. American Association of Colleges of Nursing: the voice of academic nursing. 


\section{American Journal of Pharmaceutical Education 2020; 84 (4) Article 7415.}

http://www.aacn.nche.edu/news/articles/2015/prescription-drugabuse. Accessed July 23, 2019.

9. The American Nurses Association. Abuse of Prescription Drugs. https://www.nursingworld.org/practice-policy/nursing-excellence/ official-position-statements/id/abuse-of-prescription-drugs/.

Accessed March 23, 2020.

10. The White House. Fact sheet: Obama administration announces public and private sector efforts to address prescription drug abuse and heroin use. https://www.whitehouse.gov/the-press-office/2015/ 10/21/ fact-sheet-obama-administrationannounces-public-andprivate-sector. Accessed March 23, 2020.

11. American Association of Colleges of Pharmacy. Curricular Guidelines for Pharmacy: Substance Abuse and Addictive Disease. https://www.aacp.org/sites/default/files/Curricular\% 20Guidelines $\% 20$ for $\% 20$ Pharmacy $\% 20$-\%20Substance $\%$ 20Abuse \%20and\%20Addictive\%20Disease.pdf. Accessed March 23, 2020.

12. American Association of Colleges of Nursing. AACN's Response: The Opioid Epidemic. http://www.aacnnursing.org/Portals/42/Policy/ PDF/AACN-Opioids.pdf. Accessed March 23, 2020.

13. Muzyk AJ, Peedin E, Lipetzky J, Parker H, Mceachern MP, Thomas K. Substance use education in US schools of pharmacy: A systematic review of the literature. Subst Abus. 2017;38(4): 455-463.

14. Smothers Z, Reynolds V, Mceachern M, Derouin AL, Carter BM, Muzyk A. Substance use education in schools of nursing. Nurse Educ. 2017:1.
15. Reed DA, Cook DA, Beckman TJ, Levine RB, Kern DE, Wright SM. Association between funding and quality of published medical education research. JAMA. 2007;298(9):1002-1009.

16. Kirkpatrick D. Great Ideas Revisited. Training and Development. 1996;50:54-59.

17. Muzyk A, Mullan P, Andolsek K, Smothers Z, Sanders C, Holmer S. (in press). An interprofessional substance use disorders course to improve students' educational outcomes and patients' treatment decisions. Aca Med.

18. Chappel JN, Veach TL, Krug RS. The substance abuse attitude survey: an instrument for measuring attitudes. J Stud Alcohol. 1985;46(1):48-52.

19. Dominguez DG, Fike DS, Maclaughlin EJ, Zorek JA. A comparison of the validity of two instruments assessing health professional student perceptions of interprofessional education and practice. J Interprof Care. 2014;29(2):144-149.

20. Goedhart H. The retrospective pretest and the role of pretest information in evaluative studies. Psychological Reports. 1992;70(3):699.

21. Lane C, Huws-Thomas M, Hood K, Rollnick S, Edwards K, Robling M. Measuring adaptations of motivational interviewing: the development and validation of the behavior change counseling index (BECCI). Patient Educ Counsel. 2005;56(2):166-173.

22. Harris PA, Taylor R, Thielke R, Payne J, Gonzalez N, Conde JG. Research electronic data capture (REDCap)--A metadata-driven methodology and workflow process for providing translational research informatics support. J Biomed Inform. 2009;42(2):377-378. 